\title{
An analytical solution to the extended mild-slope equation for long waves propagating over an axi-symmetric pit
}

\author{
Tae-Hwa Jung ${ }^{\text {a }}$, Kyung-Duck Suh ${ }^{\text {b,* }}$ \\ ${ }^{a}$ Department of Civil and Environmental Engineering, Seoul National University, San \\ 56-1, Shillim-dong, Gwanak-gu, Seoul 151-744, Republic of Korea \\ ${ }^{\mathrm{b}}$ Department of Civil and Environmental Engineering \& Engineering Research Institute, \\ Seoul National University, San 56-1, Shillim-dong, Gwanak-gu, Seoul 151-744, \\ Republic of Korea
}

\begin{abstract}
An analytic solution to the extended mild-slope equation is derived for long waves propagating over an axi-symmetric pit, where the water depth decreases in proportion to a power of radial distance from the pit center. The solution is obtained using the method of separation of variables and the method of Frobenius. By comparing the extended and conventional mild-slope equations for waves propagating over conical pits with different bottom slopes, it is shown that for long waves the conventional mild-slope equation is reasonably accurate for bottom slopes less than 1:3 in horizontal twodimensional domains. The effects of the pit shape on wave scattering are discussed based on the analytic solutions for different powers. Comparison is also made with an analytic solution for a cylindrical pit with a vertical sidewall. Finally, wave attenuation in the region over the pit is discussed.
\end{abstract}

Keywords: Long waves; Analytic solution; Extended mild-slope equation; Axisymmetric pit

\footnotetext{
* Corresponding author. Fax: +82-2-887-0349. E-mail addresses: togye176@ snu.ac.kr (T.-H. Jung), kdsuh@snu.ac.kr (K.-D.Suh)
} 


\section{Introduction}

Wave transformation in a nearshore area is of primary importance to provide the coastal engineers with the input wave condition for design of coastal structures and beaches. Numerical models are frequently used to calculate the transformation of waves. However, since numerical solution techniques inherently involve approximations, it is necessary to test the models against both analytic solutions and laboratory and field data from representative cases. The most rigorous test cases would involve comparisons with laboratory and field data, because they are the physical systems of interest. However, it is difficult to measure all the necessary boundary and forcing conditions, especially in field experiments. Comprehensive measurements are somewhat easier to obtain (and repeat) in a laboratory test, yet difficulties arise when trying to reproduce the laboratory wave generating and absorbing systems in numerical models. Also, experimental data always contain a certain amount of measurement errors.

Analytic solutions are another avenue for testing numerical models. Although analytic solutions are available only for special situations, they are generally developed at reduced cost, time, and labor in comparison to experiments, and comparisons with analytic solutions are a direct test of the numerical model scheme under idealized conditions. In addition, it is often simpler to use the analytic solution as a basis for evaluating the influence of specific forcing or boundary conditions on the problem.

A frequently considered problem in analytic studies of wave transformation is the long wave motion around a circular island mounted on an axi-symmetric shoal. Homma [5], Vastano and Reid [15], Jonsson et al. [7], and Zhu and Zhang [18] studied long waves around a circular island mounted on a parabolic or conical shoal. Also, Zhang and Zhu [17] and Fujima et al. [4] presented the solution around a conical island or over a parabolic shoal. Recently, Yu and Zhang [16] presented a more general solution by describing the radial bathymetry of the shoal by a power of the radial distance. More recently, Liu et al. [8] extended the Homma's solution to intermediate depth water waves by using Hunt's [6] approximate direct solution of the implicit wave dispersion equation to explicitly express the coefficients of the mild slope equation.

On the other hand, Suh et al. [13] presented the analytic solution for long waves 
propagating over a parabolic pit, in which the water depth varies in proportion to the second power of the radial distance from the pit center. The present study is to extend the Suh et al.'s solution by easing the restriction on bathymetry. First, it is extended to a pit with a different shape by describing the radial bathymetry of the pit by a different power of the radial distance; the first power corresponds to a conical pit, and the pit approaches to a cylindrical pit as the power increases. Second, in order to resolve the effects of rapidly varying bathymetry associated with the increase of the power, the extended mild-slope equation (EMSE) is used, which includes additional terms proportional to bottom curvature and square of bottom slope compared to the conventional mild-slope equation (MSE).

In the following section, we derive an analytic solution to the EMSE for long waves propagating over an axi-symmetric pit using the method of separation of variables and the method of Frobenius. The convergence of the solution is then examined with respect to the number of terms in the eigenfunction series and the Frobenius series. The accuracy of the mild-slope equation with respect to bottom slope in horizontal twodimensional domains is examined by comparing the extended and conventional mildslope equations for waves propagating over conical pits with different bottom slopes. We also discuss the effects of the pit shape on wave scattering using our analytic solution. Finally, wave attenuation in the region over the pit is discussed, and then we summarize the main conclusions.

\section{Analytic solution}

Consider an axi-symmetric pit situated in an otherwise constant depth region as shown in Fig. 1, where the origin of the horizontal coordinate system is taken to be the center of the pit, $r$ is the radial distance from the origin, and $\theta$ is the angle measured counterclockwise from the positive $x$-axis. The incident wave is assumed to be a longcrested wave propagating in the positive $x$ direction. The water depths at the origin and in the constant depth region are denoted by $h_{0}$ and $h_{1}$, respectively. The water depth in the pit is assumed to decrease from the center to the edge, according to the equation, $h=h_{0}\left(1-r^{\alpha} / a^{\alpha}\right)$, where $a$ is the radial distance from the pit center to the 
imaginary edge of the pit extended to the water surface and the power $\alpha$ is a positive integer. Denoting the radial distance to the actual edge of the pit as $b$, the water depth is given by

$$
h= \begin{cases}h_{0}\left(1-\frac{r^{\alpha}}{a^{\alpha}}\right) & r<b \\ h_{1}=h_{0}\left(1-\frac{b^{\alpha}}{a^{\alpha}}\right) & r \geq b\end{cases}
$$

Fig. 2 shows the water depth along the $x$-axis for various values of $\alpha$. The pit has a conical shape for $\alpha=1$, and the pit approaches to a cylindrical pit with a vertical side slope as $\alpha$ increases.

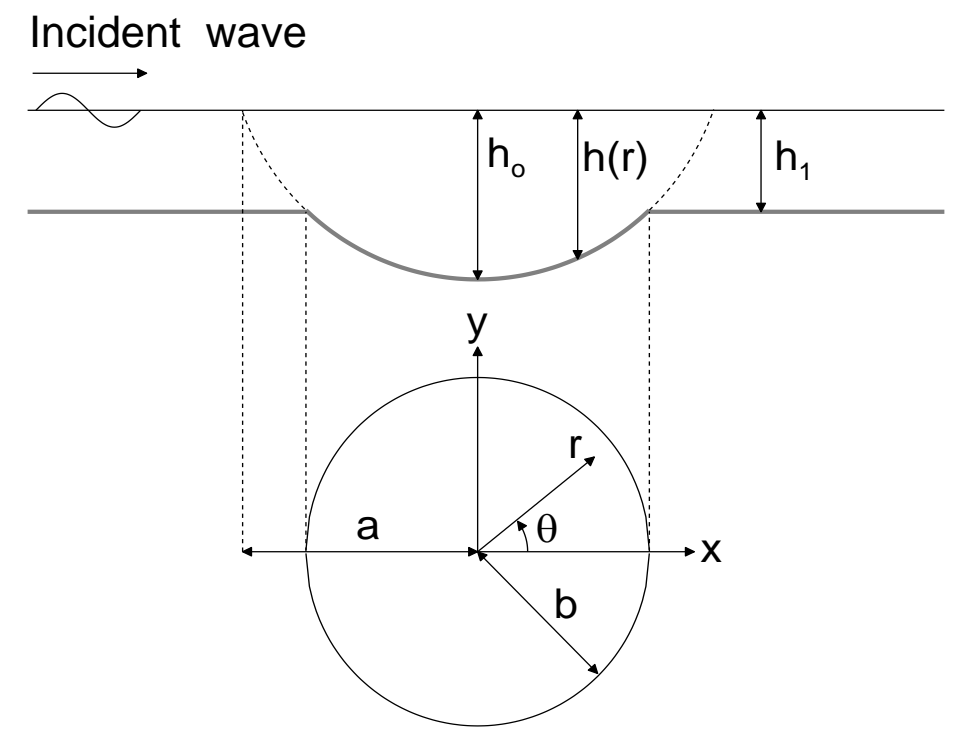

Fig. 1. Definition sketch of an axi-symmetric pit located in an otherwise constant depth region. 


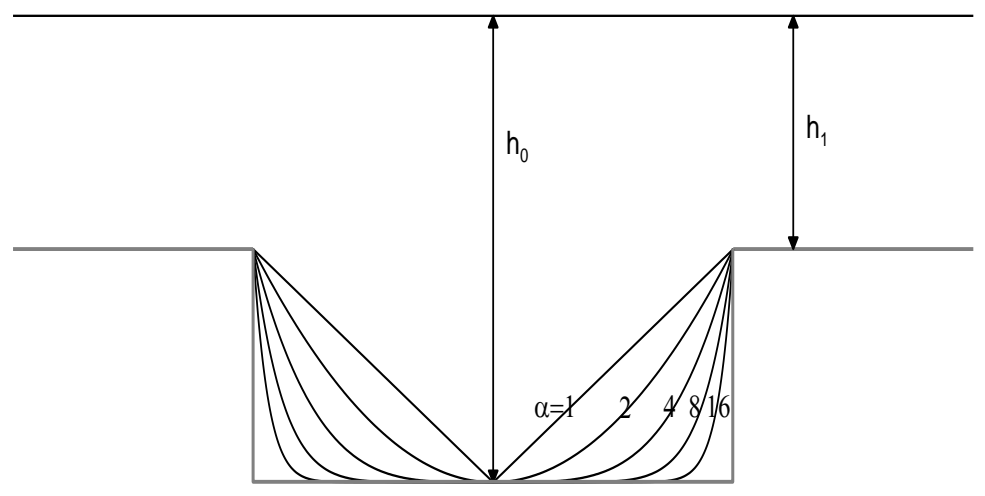

Fig. 2. Cross-sectional views along the $x$-axis of axi-symmetric pits of various values of $\alpha$.

EMSE's have been developed by Massel [10], Chamberlain and Porter [2], Suh at al. [14], and Chandrasekera and Cheung [3], all being equivalent for a monochromatic wave without evanescent modes. Among these, the second and fourth papers present simpler forms of the coefficients of the bottom curvature and slope-squared terms. In this study, we use the EMSE of Chandrasekera and Cheung given by

$\nabla \cdot\left(C C_{g} \nabla \eta\right)+\left[\omega^{2} \frac{C_{g}}{C}+g f_{1}(k h) \nabla^{2} h+g k f_{2}(k h)(\nabla h)^{2}\right] \eta=0$

where $\eta$ is the complex water surface elevation, $C$ the phase speed, $C_{g}$ the group velocity, $k$ the wave number, $\omega$ the wave angular frequency, $g$ the gravitational acceleration, $\nabla$ the horizontal gradient operator, and $f_{1}(k h)$ and $f_{2}(k h)$ are the coefficients of the curvature and slope-squared terms given, respectively, by

$$
\begin{aligned}
& f_{1}(k h)=\frac{-4 k h \cosh (k h)+\sinh (3 k h)+\sinh (k h)+8(k h)^{2} \sinh (k h)}{8 \cosh ^{3}(k h)[2 k h+\sinh (2 k h)]}-\frac{k h}{2} \frac{\tanh (k h)}{\cosh ^{2}(k h)} \\
& f_{2}(k h)=\frac{\operatorname{sech}^{2}(k h)}{6\left[2 k h+\sinh ^{2}(2 k h)\right]^{3}} \cdot\left\{8(k h)^{4}+16(k h)^{3} \sinh (2 k h)-9 \sinh ^{2}(2 k h) \cosh (2 k h)\right. \\
& \left.\quad+12(k h)\left[1+2 \sinh ^{4}(k h)\right][k h+\sinh (2 k h)]\right\}
\end{aligned}
$$


Without the last two terms in the bracket of Eq. (2), it reduces to the conventional MSE. For long (shallow water) waves, $C \cong C_{g} \cong \sqrt{g h}$ and $\omega^{2} \cong g k^{2} h$, so that Eq. (2) becomes

$$
h \nabla^{2} \eta+\nabla h \cdot \nabla \eta+\left[\frac{\omega^{2}}{g}+f_{1}(k h) \nabla^{2} h+f_{2}(k h)(\nabla h)^{2} k\right] \eta=0
$$

In principle, the functions $f_{1}(k h)$ and $f_{2}(k h)$ could be expanded as power series of $k h$. In the limit of shallow water, they show behaviors of even and odd functions, respectively, as shown in Fig. 3. These functional behaviors can also be shown by their asymptotic forms as $k h$ approaches zero. By using the first two terms in each of the Taylor series expansions of the hyperbolic functions for small $k h$ and taking the leading order terms in the numerator and denominator of Eqs. (3) and (4), we obtain $f_{1}(k h)=-(1 / 6)(k h)^{2}$ and $f_{2}(k h)=-(1 / 6) k h$, which are even and odd functions, respectively. Therefore, we expand $f_{1}(k h)$ and $f_{2}(k h)$ as

$$
\begin{aligned}
& f_{1}(k h)=a_{0}+a_{1}(k h)^{2}+a_{2}(k h)^{4}+\cdots \\
& f_{2}(k h)=b_{0}+b_{1}(k h)+b_{2}(k h)^{3}+\cdots
\end{aligned}
$$

with $b_{0}=0.0$. Keeping the first three terms of each function and determining the coefficients by the least-square method, we get $a_{0}=0.0, a_{1}=-0.16636$, $a_{2}=0.14428, b_{0}=0.0, b_{1}=-0.16640$, and $b_{2}=0.19259$. Fig. 3 shows comparisons between the exact and approximate functions of $f_{1}(k h)$ and $f_{2}(k h)$. The difference is unnoticeable within the limit of long waves, i.e., $k h<\pi / 10$, so we use the approximate functions with the first three terms in the following derivation. Now Eq. (5) can be approximated as 

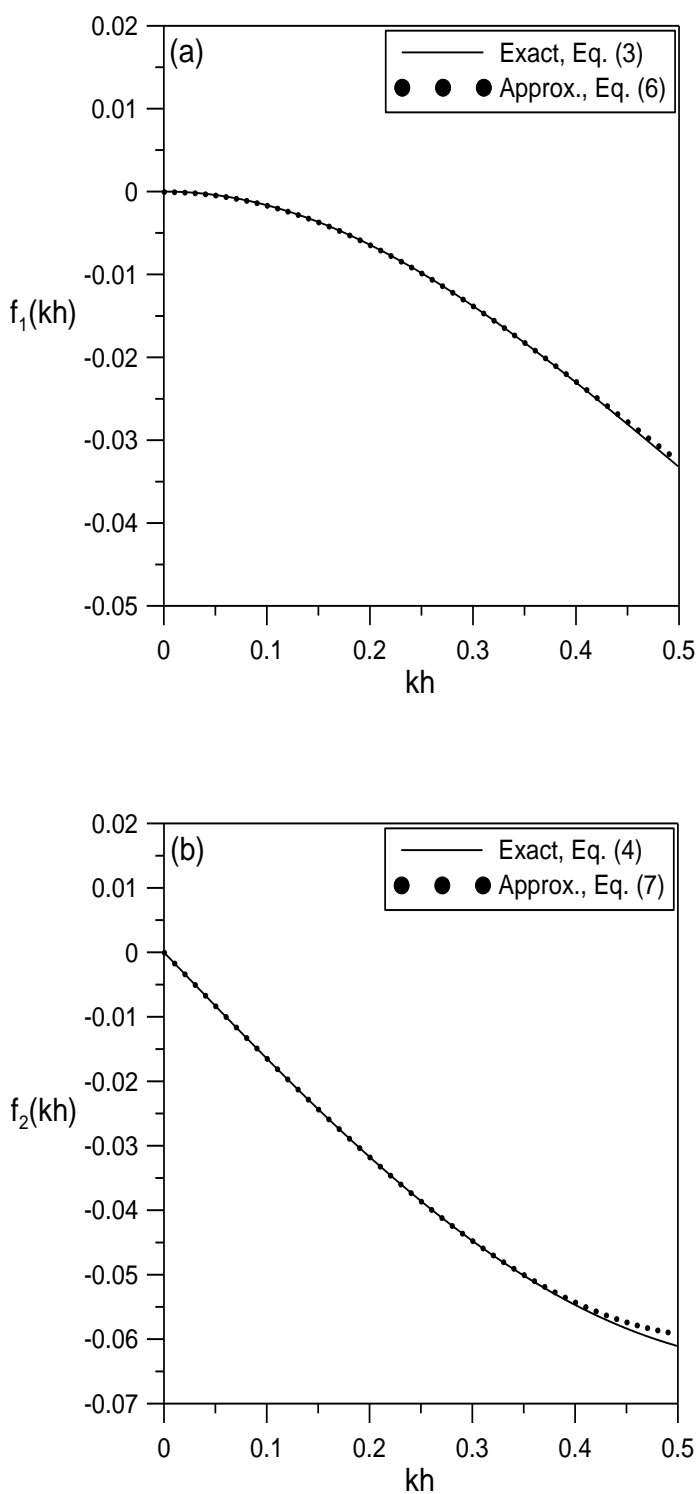

Fig. 3. Comparison between exact and approximate functions of $f_{1}(k h)$ and $f_{2}(k h)$.

$h \nabla^{2} \eta+\nabla h \cdot \nabla \eta+\frac{\omega^{2}}{g}\left[1+h\left\{a_{1}+a_{2} \frac{\omega^{2} h}{g}\right\} \nabla^{2} h+\left\{b_{1}+b_{2} \frac{\omega^{2} h}{g}\right\}(\nabla h)^{2}\right] \eta=0$

In the pit area where $h=h(r)$, this equation can be expressed in polar coordinates as 


$$
\begin{aligned}
& h\left(\frac{\partial^{2} \eta}{\partial r^{2}}+\frac{1}{r} \frac{\partial \eta}{\partial r}+\frac{1}{r^{2}} \frac{\partial^{2} \eta}{\partial \theta^{2}}\right)+\frac{d h}{d r} \frac{\partial \eta}{\partial r} \\
& +\frac{\omega^{2}}{g}\left[1+h\left\{a_{1}+a_{2} \frac{\omega^{2} h}{g}\right\} \nabla^{2} h+\left\{b_{1}+b_{2} \frac{\omega^{2} h}{g}\right\}(\nabla h)^{2}\right] \eta=0
\end{aligned}
$$

Using the method of separation of variables, i.e., by assuming $\eta$ as a product form:

$$
\eta(r, \theta)=R(r) \Theta(\theta)
$$

we obtain an eigenvalue problem for $\Theta$, which leads to

$$
\Theta_{n}(\theta)=C_{1 n} \cos n \theta+C_{2 n} \sin n \theta \quad(n=0,1,2, \cdots)
$$

where $C_{1 n}$ and $C_{2 n}$ are arbitrary constants. The function $R(r)$ corresponding to each eigenvalue $n$ can then be shown to satisfy the following ordinary differential equation:

$$
\left(a^{\alpha}-r^{\alpha}\right) r^{2} \frac{d^{2} R_{n}}{d r^{2}}+\left(a^{\alpha} r-(\alpha+1) r^{\alpha+1}\right) \frac{d R_{n}}{d r}+\left(-n^{2} a^{\alpha}+Q r^{2}+\sum_{i=1}^{3} P_{i} r^{i \alpha}\right) R_{n}=0
$$

where $Q=0$ if $\alpha<3, Q=\mu / h_{*}^{2}$ with $h_{*}=h_{0} / a^{\alpha}$ and $\mu=\omega^{2} h_{*} / g$ if $\alpha \geq 3$, and $P_{i}$ 's are given in Table 1 for different values of $\alpha$.

Since Eq. (12) is a second-order ordinary differential equation with variable coefficients, Frobenius-type series solution could be sought. It is noted that the point $r=0$ is a regular singular point of Eq. (12), and $r=a$ is an irregular singular point. According to Frobenius theory, if $R_{n}(r)$ is expanded at the point $r=0$, the series converges for all $r$ being in the disk $r<a$. Since the pit region, $r \leq b$, always lies within the disk $r<a$, the convergence of the solution is ensured.

Assuming the Frobenius series solution, $R_{n}(r)=\sum_{m=0}^{\infty} a_{m, n} r^{m+c}$, and substituting it into Eq. (12) give 
Table 1

Definitions of $P_{i}$ 's for different values of $\alpha$.

\begin{tabular}{|c|c|c|c|}
\hline & $\alpha=1$ & $\alpha=2$ & $\alpha \geq 3$ \\
\hline$P_{1}$ & $a_{2} \mu a+a_{3} \mu^{2} a^{2}+n^{2}$ & $\begin{array}{l}\frac{\mu}{h_{*}^{2}}+4 a_{2} \mu a^{2} \\
+4 a_{3} \mu^{2} a^{4}+n^{2}\end{array}$ & $\begin{array}{l}a_{2} \mu \alpha^{2} a^{\alpha} \\
+a_{3} \mu^{2} \alpha^{2} a^{2 \alpha}+n^{2}\end{array}$ \\
\hline$P_{2}$ & $\begin{array}{l}\frac{\mu}{h_{*}^{2}}-a_{2} \mu-2 a_{3} \mu^{2} a \\
+b_{1} \mu+b_{2} \mu^{2} a\end{array}$ & $\begin{array}{l}-4 a_{2} \mu-8 a_{3} \mu^{2} a^{2} \\
+4 b_{1} \mu+4 b_{2} \mu^{2} a^{2}\end{array}$ & $\begin{array}{l}-a_{2} \mu \alpha^{2}-2 a_{3} \mu^{2} \alpha^{2} a^{\alpha} \\
+b_{1} \mu \alpha^{2}+b_{2} \mu^{2} \alpha^{2} a^{\alpha}\end{array}$ \\
\hline$P_{3}$ & $a_{3} \mu^{2}-b_{2} \mu^{2}$ & $4 a_{3} \mu^{2}-4 b_{2} \mu^{2}$ & $a_{3} \mu^{2} \alpha^{2}-b_{2} \mu^{2} \alpha^{2}$ \\
\hline
\end{tabular}

$$
\begin{aligned}
& \sum_{m=0}^{\infty}\left(a^{\alpha}(m+c)(m+c-1) a_{m, n} r^{m+c}-(m+c)(m+c-1) a_{m, n} r^{m+c+\alpha}\right) \\
& +\sum_{m=0}^{\infty}\left(a^{\alpha}(m+c) a_{m, n} r^{m+c}-(\alpha+1)(m+c) a_{m, n} r^{m+c+\alpha}\right) \\
& +\sum_{m=0}^{\infty}\left(-n^{2} a^{\alpha} a_{m, n} r^{m+c}+Q a_{m, n} r^{m+c+2}+P_{1} a_{m, n} r^{m+c+\alpha}+P_{2} a_{m, n} r^{m+c+2 \alpha}+P_{3} a_{m, n} r^{m+c+3 \alpha}\right)=0
\end{aligned}
$$

Comparing the coefficients of the term of $r^{m}$, we obtain $c= \pm n$, which, in turn, gives two linearly independent solutions:

$$
\begin{aligned}
& R_{n, 1}=\sum_{m=0}^{\infty} \beta_{m, n} r^{m+n} \\
& R_{n, 2}=R_{n, 1} \ln r+\sum_{m=0}^{\infty} \gamma_{m, n} r^{m-n}
\end{aligned}
$$

Imposing the condition that water surface elevation must be finite at the origin, $R_{n, 2}$ can be omitted. Substituting Eq. (14) into Eq. (13), we obtain the coefficients $\beta_{m, n}$ as follows for different values of $\alpha$.

In the case of $\alpha=1, \beta_{0, n}$ is an arbitrary constant, and 


$$
\begin{array}{ll}
\beta_{m, n}=\frac{\left((m+n-1)(m+n)-P_{1}\right) \beta_{m-1, n}}{a m(m+2 n)} & m=1 \\
\beta_{m, n}=\frac{\left((m+n-1)(m+n)-P_{1}\right) \beta_{m-1, n}-P_{2} \beta_{m-2, n}}{a m(m+2 n)} & m=2 \\
\beta_{m, n}=\frac{\left((m+n-1)(m+n)-P_{1}\right) \beta_{m-1, n}-P_{2} \beta_{m-2, n}-P_{3} \beta_{m-3, n}}{a m(m+2 n)} & m \geq 3
\end{array}
$$

In the case of $\alpha=2, \beta_{0, n}$ is an arbitrary constant, and

$$
\begin{array}{ll}
\beta_{m, n}=\frac{\left((m+n-2)(m+n)-P_{1}\right) \beta_{m-2, n}}{a^{2} m(m+2 n)} & m=2 \\
\beta_{m, n}=\frac{\left((m+n-2)(m+n)-P_{1}\right) \beta_{m-2, n}-P_{2} \beta_{m-4, n}}{a^{2} m(m+2 n)} & m=4 \\
\beta_{m, n}=\frac{\left((m+n-2)(m+n)-P_{1}\right) \beta_{m-2, n}-P_{2} \beta_{m-4, n}-P_{3} \beta_{m-6, n}}{a^{2} m(m+2 n)} & m \geq 6
\end{array}
$$

In the case of $\alpha \geq 3, \beta_{0, n}$ is an arbitrary constant, and

$$
\begin{array}{ll}
\beta_{m, n}=0 & m=1 \\
\beta_{m, n}=\frac{-\frac{\mu}{h_{*}^{2}} \beta_{m-2, n}}{a^{\alpha}(m+2 n)(m)} & 2 \leq m<\alpha \\
\beta_{m, n}=\frac{\left((m-\alpha+n)(m+n)-P_{1}\right) \beta_{m-\alpha, n}-\frac{\mu}{h_{*}^{2}} \beta_{m-2, n}}{a^{\alpha}(m+2 n)(m)} & \alpha \leq m<2 \alpha \\
\beta_{m, n}=\frac{\left((m-\alpha+n)(m+n)-P_{1}\right) \beta_{m-\alpha, n}-\frac{\mu}{h_{*}^{2}} \beta_{m-2, n}-P_{2} \beta_{m-2 \alpha, n}}{a^{\alpha}(m+2 n)(m)} & 2 \alpha \leq m<3 \alpha \\
\beta_{m, n}=\frac{\left((m-\alpha+n)(m+n)-P_{1}\right) a_{m-\alpha, n}-\frac{\mu}{h_{*}^{2}} \beta_{m-2, n}-P_{2} \beta_{m-2 \alpha, n}-P_{3} \beta_{m-3 \alpha, n}}{a^{\alpha}(m+2 n)(m)} & m \geq 3 \alpha
\end{array}
$$

Finally, for long waves over an axi-symmetric pit, the water surface elevation is given by 
$\eta=\sum_{n=0}^{\infty} A_{n} R_{n}\left(C_{1 n} \cos n \theta+C_{2 n} \sin n \theta\right)$

where $A_{n}$ is an arbitrary constant.

The long-crested incident wave propagating in the positive $x$ direction can be represented by

$\eta_{0}=a_{i} e^{i k x}$

where $a_{i}$ is the incident wave amplitude and $i=\sqrt{-1}$. It is known that $\eta_{0}$ can be expanded into

$\eta_{0}=a_{i} \sum_{n=0}^{\infty} i^{n} \varepsilon_{n} J_{n}(k r) \cos n \theta$

where $J_{n}$ is the Bessel function of the first kind of order $n$, and the Jacobi symbols $\varepsilon_{n}=1$ for $n=0$ and $\varepsilon_{n}=2$ for $n \geq 1$, respectively.

In order to obtain the full solution, we apply the method of matched eigen-expansions. Accordingly, we divide the fluid domain into two regions in the horizontal plane: the finite region with variable depth $(r<b)$, and the semi-infinite far region with constant depth $(r \geq b)$. In the far region, the general solution of the complex surface elevation satisfies the Sommerfeld radiation condition at infinity as well as the symmetry condition about the $x$ axis and can be written as

$\eta_{1}=\eta_{0}+\sum_{n=0}^{\infty} D_{n} H_{n}^{(1)}(k r) \cos n \theta, \quad r \geq b$

where $D_{n}$ is a set of complex constants to be determined, and $H_{n}^{(1)}$ is the Hankel function of the first kind of order $n$.

In the finite region with varying depth, the water surface elevation can be written as 
follows:

$\eta_{2}=\sum_{n=0}^{\infty} B_{n} R_{n} \cos n \theta$

where $B_{n}=A_{n} C_{1 n}$ is again a set of complex constants to be determined. The terms associated with $\sin n \theta$ have been dropped based on the symmetry condition.

At $r=b$ where the slope of bottom is discontinuous, the continuity of surface elevation and mass flow conservation require (Porter and Staziker [11])

$$
\begin{array}{ll}
\eta_{1}=\eta_{2} & \text { at } r=b \\
f_{1} \eta_{1}\left(\frac{\partial h_{2}}{\partial r}-\frac{\partial h_{1}}{\partial r}\right)+f_{0}\left(\frac{\partial \eta_{2}}{\partial r}-\frac{\partial \eta_{1}}{\partial r}\right)=0 & \text { at } r=b
\end{array}
$$

respectively, where

$$
f_{0}=\frac{C C_{g}}{g}
$$

and $h_{1}$ and $h_{2}$ are the water depths in the regions of $r \geq b$ and $r<b$, respectively.

Substituting Eqs. (30) and (31) into Eqs. (32) and (33) while noting that $\{\cos n \theta\}$ form an orthogonal set, we have

$$
\begin{aligned}
& B_{n} R_{n}(b)-D_{n} H_{n}^{(1)}(k b)=a_{i} i^{n} \varepsilon_{n} J_{n}(k b) \\
& B_{n}\left[-f_{1} R_{n}(b) h_{0} \alpha \frac{b^{\alpha-1}}{a^{\alpha}}+f_{0} R_{n}{ }^{\prime}(b)\right]-D_{n}\left[f_{0} k H_{n}^{(1) '}(k b)\right]=f_{0} a_{i} k i^{n} \varepsilon_{n} J_{n}{ }^{\prime}(k b)
\end{aligned}
$$

where the prime denotes derivatives. Solving for $B_{n}$ and $D_{n}$, we find 


$$
\begin{aligned}
& B_{n}=a_{i} k i^{n} \varepsilon_{n} \frac{f_{0} H_{n}^{(1) '}(k b) J_{n}(k b)-H_{n}^{(1)}(k b) f_{0} J_{n}{ }^{\prime}(k b)}{R_{n}(b) f_{0} k H_{n}^{(1) '}(k b)-H_{n}^{(1)}(k b)\left[-f_{1} R_{n}(b) h_{0} \alpha b^{\alpha-1} / a^{\alpha}+f_{0} R_{n}{ }^{\prime}(b)\right]} \\
& D_{n}=a_{i} i^{n} \varepsilon_{n} \frac{-R_{n}(b) f_{0} k J_{n}{ }^{\prime}(k b)+\left[-f_{1} R_{n}(b) h_{0} \alpha b^{\alpha-1} / a^{\alpha}+f_{0} R_{n}{ }^{\prime}(b)\right] J_{n}(k b)}{R_{n}(b) f_{0} k H_{n}^{(1)}{ }^{\prime}(k b)-H_{n}^{(1)}(k b)\left[-f_{1} R_{n}(b) h_{0} \alpha b^{\alpha-1} / a^{\alpha}+f_{0} R_{n}{ }^{\prime}(b)\right]}
\end{aligned}
$$

Substituting these coefficients back into Eqs. (30) and (31), we can compute the water surface elevation for the whole domain.

\section{Results and discussions}

\subsection{Convergence of solution}

The analytic solution for $\eta$ involves an infinite series, but in practice this must be properly truncated. In other words, we must use an integer $N$ that is large enough such that the infinite series in Eqs. (29), (30) and (31) are approximated with the desired accuracy. The number of terms, $M$, of the truncated Frobenius series of Eq. (14) should also be large enough to give accurate results. Numerical tests showed that inside the region of the pit, a required value of $N$ increases with $r$, but the results show little difference for $N \geq 6$. Outside the region of the pit, however, a larger $N$ was necessary as leaving apart from the pit to calculate correct incident waves. It was also shown that the value of $M$ required for the convergence of the Frobenius series increases with both the radial distance $r$ and the power $\alpha$. On the other hand, the required value of $N$ was little affected by $\alpha$. In this study, therefore, with $N=40$ fixed, $M$ was determined for $r=b$ and $n=40$ such that $\left|\beta_{M, 40} b^{M+40}\right| /\left|\sum_{m=0}^{M} \beta_{m, 40} b^{m+40}\right|<10^{-5}$ is satisfied. This $M$ was then used for the

calculation of $R_{n}$ or $R_{n}^{\prime}$ in Eqs. (31), (36), and (37). The Bessel functions in the analytic solution were computed using the subroutines in [12].

\subsection{Accuracy of the mild-slope equation}


Booij [1] argued that accurate predictions of wave reflection using the MSE were limited to bottom slopes of less than 1:3 by showing that the MSE solution does not agree with the exact finite element method (FEM) solution to the Laplace equation for bottom slopes greater than 1:3. Later, Suh et al. [13] found that the MSE solution does not agree with the exact FEM solution even for slopes less than 1:3. They found that this disagreement is primarily due to the slope discontinuity at both ends of the slope that can be represented by the bottom curvature term of the EMSE. They also found that when only the bottom slope is concerned the MSE is accurate up to 1:1 rather than 1:3 slope. The aforementioned arguments are based on the numerical tests in a horizontal one-dimensional domain.

In this section, we compare the analytic solutions to EMSE and MSE in horizontal two-dimensional domains. Computations were made with $\alpha=1, h_{0}=9.6 \mathrm{~m}, h_{1}=$ $3.2 \mathrm{~m}, k_{1} h_{1}=0.167$, and three different values of $b ; 3.2,6.4$, and $19.2 \mathrm{~m}$, which give constant bottom slopes of the pit of 2:1, 1:1, and 1:3, respectively. These cone-shaped pits are beneficial to test the accuracy of the wave equations with respect to the bottom slope because the slope is constant in the pits. However, the bottom curvature is not constant.

Figs. 4 to 6 show the values of the diffraction coefficients, or the wave amplitude relative to the incident amplitude, along the $x$-and $y$-axis, calculated by the EMSE, MSE, MSE with the bottom curvature term, and the MSE with the slope-squared term, for cone-shaped pits with different bottom slopes. The results are presented in terms of dimensionless coordinates, $x / L_{1}$ and $y / L_{1}$, where $L_{1}$ is the wavelength in the constant depth region. Along the $x$-axis, the spatial variations of the diffraction coefficients of all the equations are in phase for the bottom slopes of $1: 1$ and 1:3, whereas for the slope of 2:1 the result of the MSE and MSE with the slope-squared term is out of phase with others. Along the $y$-axis, the EMSE and the MSE with the bottom curvature term are in opposite phase with other two equations for the slopes of 2:1 and $1: 1$. It is interesting to note that the MSE with the slope-squared term gives greater difference from the EMSE than the MSE does. This is because the bottom curvature term in the bracket of Eq. (5) is positive but the slope-squared term is negative, everywhere inside the pit. Note that both $f_{1}(k h)$ and $f_{2}(k h)$ terms are always 
negative as shown in Fig. 3, while $\nabla^{2} h$ and $(\nabla h)^{2}$ are negative and positive, respectively, inside the pit. Therefore, the bottom curvature term and the slope-squared term behave to cancel out each other, the former dominating the latter in general.

Finally, to examine the accuracy of the MSE with respect to the bottom slope, the MSE with the bottom curvature term is compared with the EMSE, which includes the full $\left(\nabla h\right.$ and $\left.(\nabla h)^{2}\right)$ effect of bottom slope as well as the effect of bottom curvature. The comparison of these two equations would elucidate the difference of the effect of bottom slope between the MSE and EMSE because the MSE includes the effect of bottom slope of only $\nabla h$. If they show only a little difference for a certain bottom slope, we could say that the MSE is accurate up to that bottom slope. As shown in Figs. 5 and 6, the MSE with the bottom curvature term shows only reasonable agreement with the EMSE for 1:3 slope and poor agreement for 1:1 slope, which is different from the results in a horizontal one-dimensional domain where the agreement was almost perfect up to 1:1 slope. Based on these numerical tests, we could conclude that the MSE is only reasonably accurate up to the slope of 1:3 in horizontal two-dimensional domains.
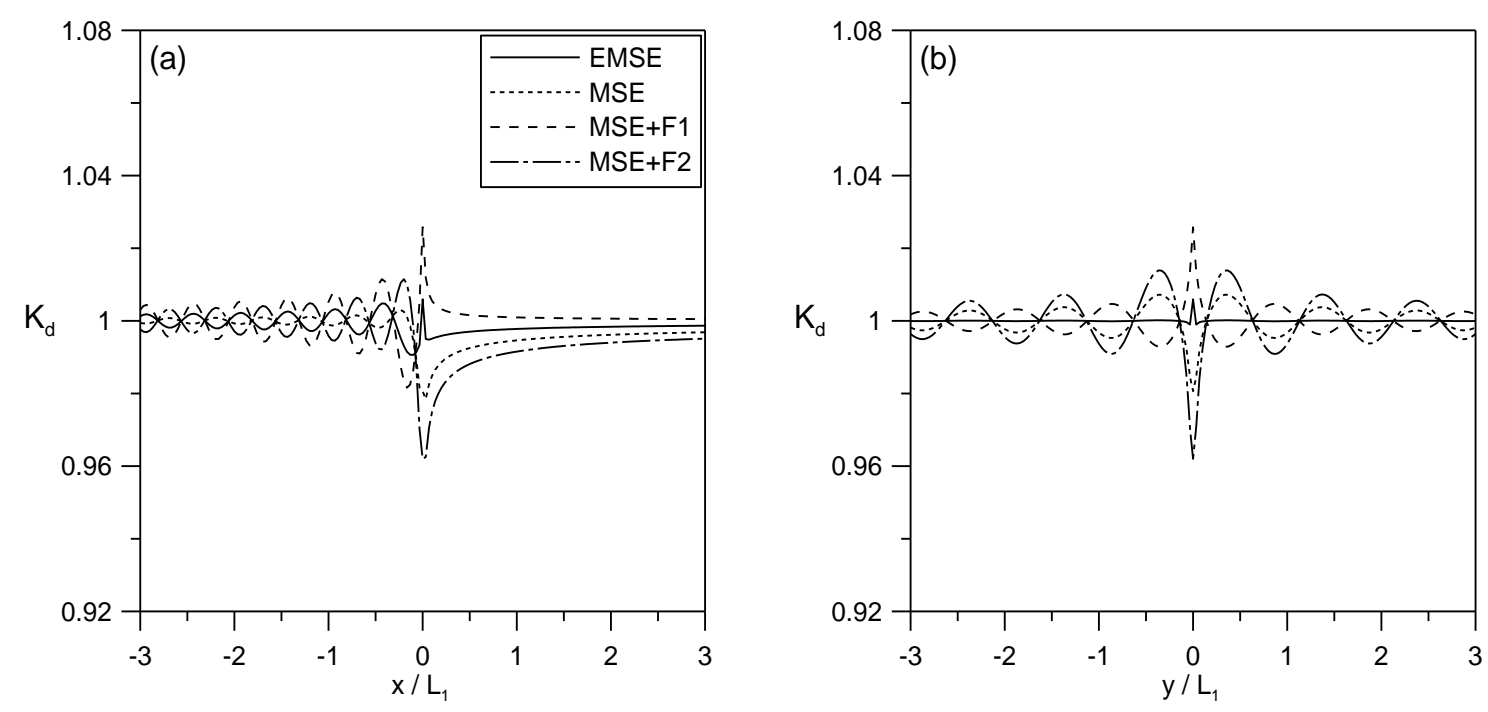

Slope $=2: 1$

Fig. 4. Diffraction coefficients along (a) $x$-axis and (b) $y$-axis for a cone-shaped pit with $h_{0}=9.6 \mathrm{~m}, h_{1}=3.2 \mathrm{~m}, k_{1} h_{1}=0.167$, and $b=3.2 \mathrm{~m}:-\longrightarrow=$ EMSE; $\cdots \cdot . \cdot$ = MSE; - - - - = MSE plus bottom curvature term; - - - = MSE plus slope-squared term. 

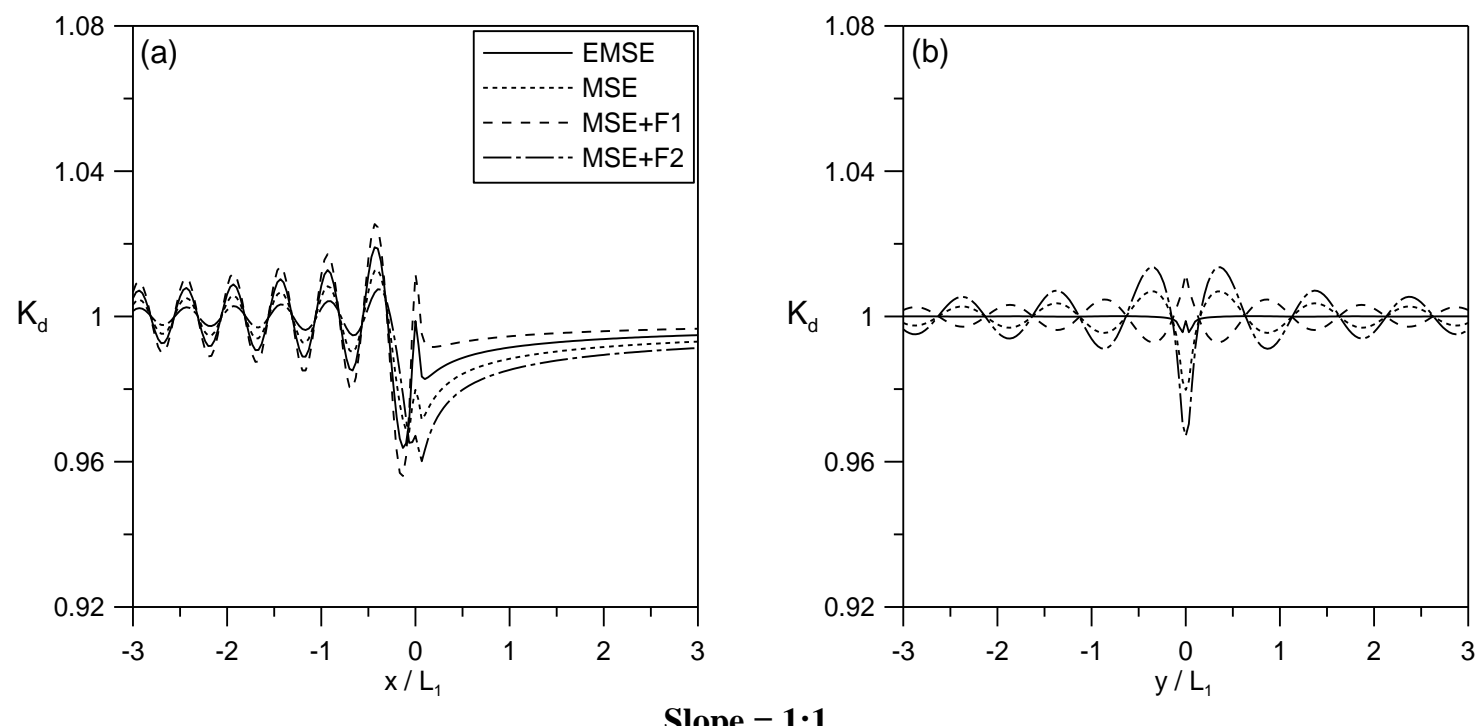

Slope $=1: 1$

Fig. 5. Same as Fig. 4 except for $b=6.4 \mathrm{~m}$.
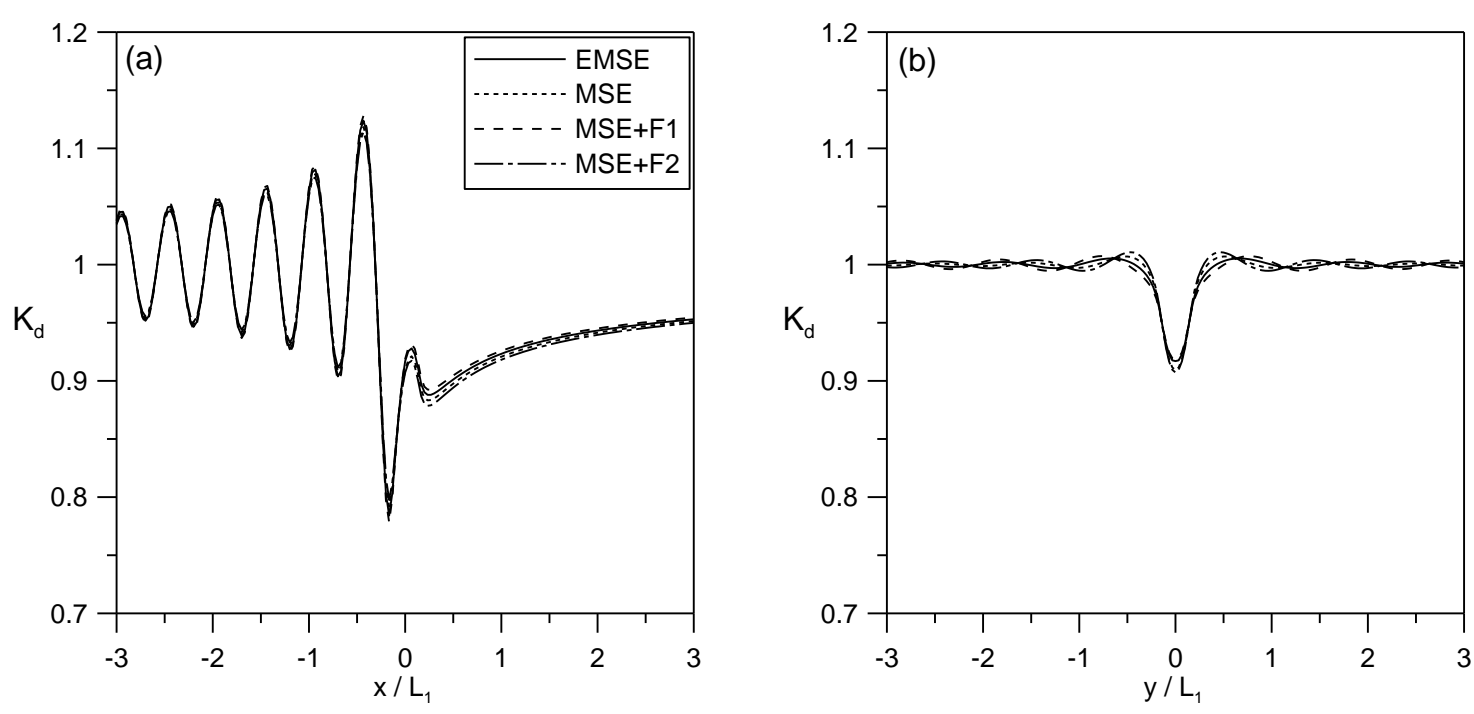

Slope $=1: 3$

Fig. 6. Same as Fig. 4 except for $b=19.2 \mathrm{~m}$.

\subsection{Effect of pit shapes}

As the power $\alpha$ increases from one to infinity, the pit changes from a conical 
shape to a cylindrical shape as shown in Fig. 2. In this section, we examine the effects of the shape of the pit based on the analytic solutions for different values of $\alpha$. Computations were made with $h_{0}=9.6 \mathrm{~m}, h_{1}=3.2 \mathrm{~m}, k_{1} h_{1}=0.167$, and $b=0.5 L_{1}$. Fig. 7 shows diffraction coefficients along the $x$ - and $y$-axis for the case of $\alpha=1,2,4,8$, and 16. As $\alpha$ increases, the partial standing wave (due to reflection) in front of the pit increases and more energy is also scattered laterally due to refraction; thus, there is more of a reduction of wave heights in the shadow zone. The location of the smallest wave height in the shadow zone is shifted backwards as $\alpha$ increases, but the location of the small peak in the pit, which is due to wave reflection from the rear wall of the pit, remains almost constant at $x / L_{1} \cong 0.4$. The lateral variation of the diffraction coefficient also increases with $\alpha$, showing the locations of its maxima and minima be shifted farther from the pit as $\alpha$ increases. It is noticeable that a new local peak starts to appear near the upwave boundary of the pit (i.e., $x / L_{1} \cong-0.4$ ) as $\alpha$ increases probably due to re-reflection of the waves from the steeper upwave slope of the pit.
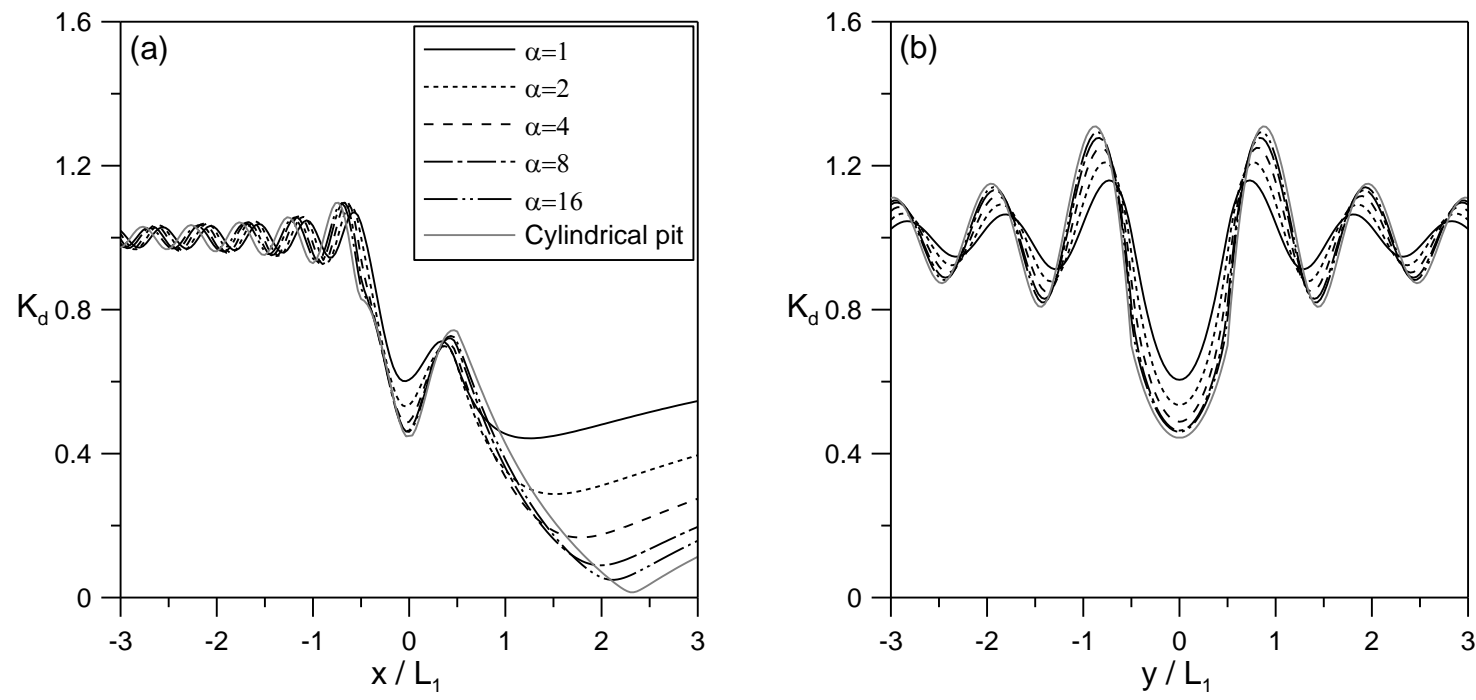

Fig. 7. Diffraction coefficients along (a) $x$-axis and (b) $y$-axis for axi-symmetric pits with different $\alpha$ 's: $h_{0}=9.6 \mathrm{~m}, h_{1}=3.2 \mathrm{~m}, k_{1} h_{1}=0.167$, and $b=0.5 L_{1}$ are used, and the Longuet-Higgins [9] solution for a cylindrical pit is also shown. 
Longuet-Higgins [9] has studied wave propagation over a submerged circular sill. His solution can be used without modification for a cylindrical pit, the depth of which can be described by Eq. (1) with $\alpha=\infty$. The Longuet-Higgins' solution for a cylindrical pit is shown in Fig. 7 along with the present solutions with different $\alpha$ 's. As expected, the present solution approaches the Longuet-Higgins' solution as $\alpha$ increases.

It would be interesting to see whether the conventional mild-slope solution would approach the Longuet-Higgins' solution as $\alpha$ increases. Without showing the result of the conventional mild-slope equation, we just mention that it is almost same as that of the extended mild-slope equation shown in Fig. 7. This might be because the mild-slope assumption is satisfied in most of the region except the very edge of the pit. The average slope of the pit, $\left(h_{0}-h_{1}\right) / b$, is about 0.11 and the effect of higher order terms is negligible in most of the region in the pit.

To visualize the wave transformation inside and around the pit, the threedimensional plot of the diffraction coefficient is given in Fig. 8 for the case of $\alpha=16$. It can be seen that in front of the pit a partial standing wave system develops, while in the lee of the pit a shadow zone exists in which wave heights are reduced.

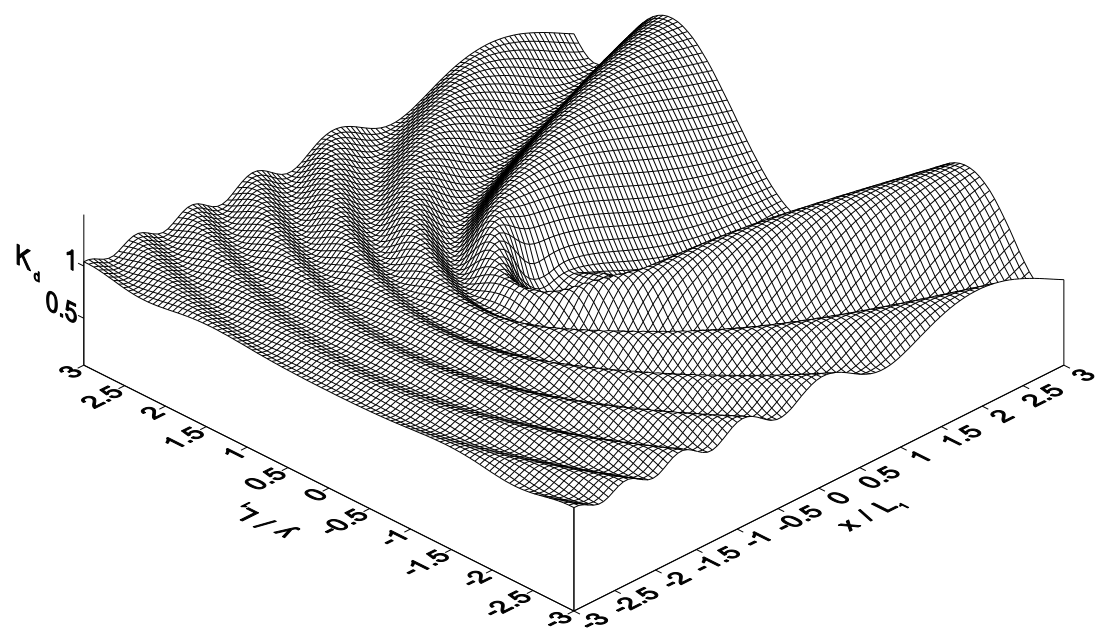

Fig. 8. Diffraction coefficients around a pit with $\alpha=16, h_{0}=9.6 \mathrm{~m}, h_{1}=3.2 \mathrm{~m}$,

$$
k_{1} h_{1}=0.167 \text {, and } b=0.5 L_{1} \text {. }
$$




\subsection{Wave attenuation inside pits}

Long waves propagating over a submerged island are trapped in the region over the island so that the amplitude of each wave mode is amplified at the resonant frequencies. On the contrary, in the case of a pit, wave attenuation occurs in such a way that the wave amplitude becomes smaller than the incident amplitude in the region over the pit. Fig. 9 shows the amplitudes of the first wave modes in the region over the pit relative to the incident amplitude as a function of the dimensionless frequency, $b \omega / \sqrt{g h_{0}}$, for different values of $\alpha$. The geometry of the pit is the same as that used to produce Fig. 7. Calculation was made up to the dimensionless frequency of 7.0 to see the behavior of the amplitudes with the change of the frequency, but the long wave approximation (i.e. $k h \leq \pi / 10)$ is satisfied only up to the dimensionless frequency of about 1.8 for the largest water depth at the center of the pit.

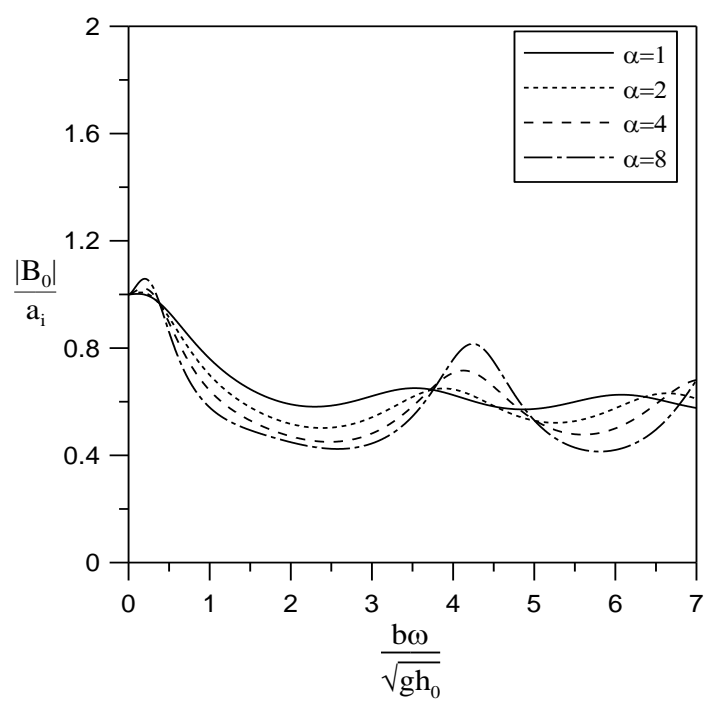

Fig. 9. Dimensionless amplitudes of the first wave modes in the region of pits with different $\alpha$ 's as a function of dimensionless frequency, $b \omega / \sqrt{g h_{0}}$.

As shown in Fig. 9, the dimensionless amplitude of the first wave mode $(n=0)$ is unity for very long waves, decreasing to the dimensionless frequency of about 2.3, and bouncing to oscillate around 0.6 for larger frequencies. The oscillation is amplified with the power, $\alpha$. On the contrary, the amplitudes of the higher modes being very small for 
very long waves increase monotonically with the frequency as shown in Fig. 10. In the case of a submerged circular sill, increasing $n$ leads to higher and sharper resonant peaks (see Longuet-Higgins [9]). In the case of a circular pit, however, the amplitudes of the higher wave modes are much smaller than that of the first wave mode. In conclusion, wave attenuation occurs in the region over the pit, as expected, with greater variation with frequency for larger $\alpha$.

It may be possible to use the present analytic solution for the wave trapping over a submerged mound by modifying the equation for water depth to $h=h_{0}\left(1+r^{\alpha} / a^{\alpha}\right)$ so as to represent a mound.

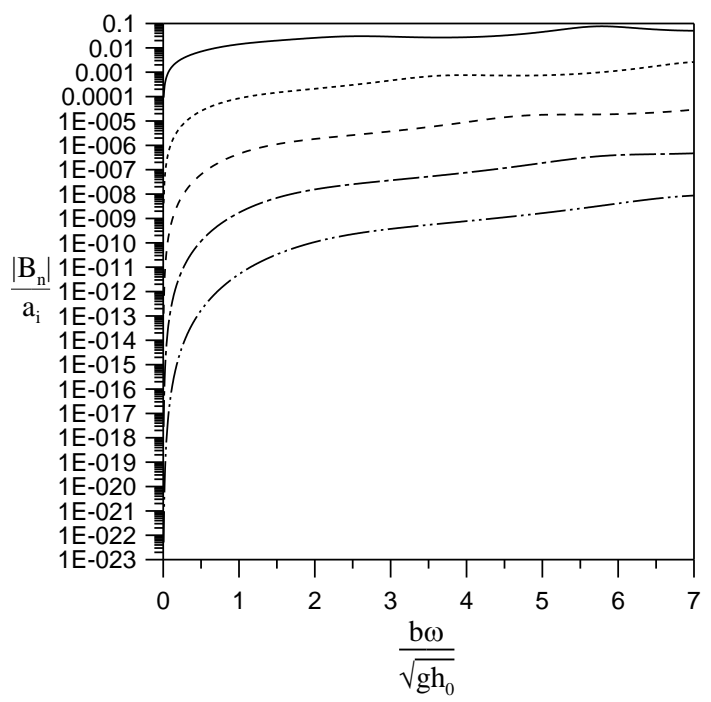

Fig. 10. Dimensionless amplitudes of the higher wave modes in the region of pits with $\alpha=8$ as a function of dimensionless frequency, $b \omega / \sqrt{g h_{0}}$.

\section{Conclusion}

We derived an approximated analytic solution to the extended mild-slope equation for long waves propagating over an axi-symmetric pit located in an otherwise constant depth region. It was found that the conventional mild-slope equation is reasonably accurate up to the bottom slope of 1:3 in horizontal two-dimensional domains, which is different from the previous finding that the mild-slope equation is accurate up to the 
slope of 1:1 in a horizontal one-dimensional domain. The effects of the power $\alpha$ were also examined, and the variation in wave scattering for different $\alpha$ 's was described. Finally, the wave attenuation in the region of the pit was described.

In the above statement regarding the accuracy of the mild-slope equation with respect to bottom slope, it should be noted that the present tests in two-dimensional domains were made for long waves, whilst the previous finding in one-dimensional domains was obtained for intermediate-depth water waves. Therefore, the statement that the mild-slope equation in a two-dimensional domain is accurate up to the bottom slope of $1: 3$ is effective only for long waves in shallow water. A further study is needed for intermediate-depth water waves. Another limitation of the statement is that the extended mild-slope equation was not proven to be more accurate than the mild-slope equation by comparing their results with a third-party result which can be considered to be exact (as Suh et al. [14] have compared the numerical solutions of original and extended mildslope equations with the finite element solution for two-dimensional Laplace equation for Booij's [1] ramp problem). We wish our solutions will be compared with an accurate third-party solution, e.g., finite element or boundary element solution for threedimensional Laplace equation, in near future.

\section{Acknowledgments}

This study was supported by the Brain Korea 21 Project. The second author was also supported by the Project for Development of Reliability Based Design Method for Port and Harbor Structures of Korea Ministry of Marine Affairs and Fisheries. The authors are grateful to the anonymous referee for suggesting the correct matching conditions at the edge of the pit.

\section{References}

[1] N. Booij, A note on the accuracy of the mild-slope equation, Coastal Eng. 7, 191203 (1983). 
[2] P.G. Chamberlain, D. Porter, The modified mild-slope equation, J. Fluid Mech., 291, 393-407 (1995).

[3] C.N. Chandrasekera, K.F. Cheung, Extended linear refraction-diffraction model, J. Waterw. Port Coast. Ocean Eng. 123, 280-296 (1997).

[4] K. Fujima, D. Yuliadi, C. Goto, K. Hayashi, T. Shigemura, Characteristics of long waves trapped by conical island, Coastal Eng. in Japan 38, 111-132 (1995).

[5] S. Homma, On the behavior of seismic sea waves around circular island, Geophys. Mag. XXI, 199-208 (1950).

[6] J.N. Hunt, Direct solution of wave dispersion equation, J. Waterw. Port Coast. Ocean Div. Proc. ASCE 105, 457-459 (1979).

[7] I.G. Jonsson, O. Skovgaard, O. Brink-Kjaer, Diffraction and refraction calculations for waves incident on an island, J. Marine Res. 34, 469-496 (1976).

[8] H.W. Liu, P.Z. Lin, N.J. Shankar, An analytical solution of the mild-slope equation for waves around a circular island on a paraboloidal shoal, Coastal Eng. 51, 421-437 (2004).

[9] M.S. Longuet-Higgins, On the trapping of wave energy round islands, J. Fluid Mech., 29, 781-821 (1967).

[10] S.R. Massel, Extended refraction-diffraction equation for surface waves, Coastal Eng. 19, 97-126 (1993).

[11] D. Porter, D.J. Staziker, Extensions of the mild-slope equation, J. Fluid Mech., 300, 367-382 (1995).

[12] W.H. Press, S.A. Teukolsky, W.T. Vetterling, B.P. Flannery, Numerical Recipes in FORTRAN: the Art of Scientific Computing, Cambridge University Press, Cambridge (1992).

[13] K.-D. Suh, T.-H. Jung, M.C. Haller, Long waves propagating over a circular bowl pit, Wave Motion 42, 143-154 (2005).

[14] K.D. Suh, C. Lee, W.S. Park, Time-dependent equations for wave propagation on rapidly varying topography, Coastal Eng. 32, 91-117 (1997).

[15] A.C. Vastano, R.O. Reid, Tsunami response for islands: Verification of a numerical procedure, J. Marine Res. 25, 129-139 (1967).

[16] X. Yu, B. Zhang, An extended analytic solution for combined refraction and diffraction of long waves over circular shoals, Ocean Eng. 30, 1253-1267 (2003). 
[17] Y. Zhang, S. Zhu, New solutions for the propagation of long water waves over variable depth, J. Fluid Mech. 278, 391-406 (1994).

[18] S. Zhu, Y. Zhang, Scattering of long waves around a circular island mounted on a conical shoal, Wave Motion 23, 353-362 (1996). 